\title{
HEXAGONAL EXTENSIONS OF TOROIDAL MAPS AND HYPERMAPS
}

\author{
MARIA ELISA FERNANDES, DIMITRI LEEMANS, AND ASIA IVIĆ WEISS
}

Dedicated to Egon Schulte on the occasion of his 60th birthday

\begin{abstract}
The rank 3 concept of a hypermap has recently been generalized to a higher rank structure in which hypermaps can be seen as "hyperfaces" but very few examples can be found in literature. We study finite rank 4 structures obtained by hexagonal extensions of toroidal hypermaps. Many new examples are produced that are regular or chiral, even when the extensions are polytopal. We also construct a new infinite family of finite nonlinear hexagonal extensions of the tetrahedron.
\end{abstract}

Keywords: Regularity, chirality, thin geometries, hypermaps, abstract polytopes 2000 Math Subj. Class: 52B11, 20 D06.

\section{INTRODUCTION}

A concept of a homogeneous honeycomb in Euclidean space was introduced by Sommerville in 1929 in his book "An Introduction to the Geometry of $n$ Dimensions" [41] as an object consisting of polyhedral cells, all alike, such that each rotation that is a symmetry operation of a cell is also a symmetry operation of the whole configuration. This definition inspired Coxeter to name a map regular whenever its group of automorphisms contains, for each of its faces, elements that cyclically permute edges of that face, and also contains automorphisms that, for each of its vertices, cyclically permute the edges meeting at the vertex. Coxeter distinguished two kinds of regular maps: reflexible and irreflexible [16]; nowadays commonly referred to respectively as regular and chiral maps (he called a regular map reflexible when the group of automorphisms of the map contains an element that fixes an edge and the two faces that contain that edge, but interchanges the two vertices of the edge, otherwise he called the map irreflexible).

Earliest known examples of chiral maps were produced by Heffter in 1898 [25] as a family of maps of Schläfli type $\left\{2^{k}-1,2^{k}-1\right\}$ for $k>2$ (the first number describes the size of the faces and the second the degrees of the vertices of the map). In the 1940's Coxeter classified regular and chiral maps on the torus [14]. In 1966, Sherk [40], a PhD student of Coxeter, looked for chiral maps of small genus and constructed an infinite family of chiral maps of type $\{6,6\}$ (with the smallest member of that family embeddable on a surface of genus 7). About the same time Edmonds - a well-known and controversial Canadian combinatorist - re-discovered, but never published, Heffter's map of type $\{7,7\}$ (also on a surface of genus 7 ). In 1969, Garbe [22] enumerated all regular maps on orientable surfaces of genus 2, 3, 4, 5 and 6 , and proved that there are no chiral maps among them. A number of papers appeared thereafter dealing with chiral maps. A first systematic search for regular and chiral maps of higher genus was conducted by Conder and Dobcsányi [9] 
and resulted in the complete list of regular and chiral maps on surfaces of genus 2 to 15 in the orientable case and regular maps on surfaces of genus 3 to 30 in the non-orientable case. Subsequently, Conder expanded this list several times to include maps of increasingly higher genus. It now contains maps up to genus 301 in the orientable case and up to genus 602 in the non-orientable case [11].

In 1970 [15], Coxeter extended the notion of a chiral map by introducing the concept of a twisted honeycomb, a finite abstract object or rank 4 derived from a honeycomb, which is chiral in a sense that it inherits all the rotations of its cells but not its reflections. Two similar examples of such structures, both with only one polyhedral cell, were described earlier by Weber and Seifert in 1933 [45]. Coxeter produced a number of non-trivial examples, which he constructed from 3dimensional euclidean, spherical and hyperbolic tessellations with spherical facets and vertex-figures, by looking at their Petrie polygons which naturally occur, in left- and right-handed varieties (each such polygon has three, but not four, consecutive edges belonging to a cell). Identifying vertices of each such, say left-handed Petrie polygon, that are separated by a fixed number of edges he observed that the resulting object may be regular (or as he called it reflexible) or may have the vertices on its right-handed Petrie polygons separated by a different number of edges in which case he called such a rank 4 object twisted. Twisted honeycomb is not symmetrical by a "reflection" in a sense that its automorphism group contains no involution that fixes, for example, a rank 2 face of the object but interchanges the two cells sharing this rank 2 face. The concept of twisted honeycomb inspired the modern definition of a chiral polytope, an abstract object of any rank that is maximally symmetric by abstract rotations but never by an abstract reflection (see Section 2).

The twisted honeycombs are finite structures resembling classical polytopes combinatorially in the sense that their facets and vertex-figures are spherical. In 1977 Coxeter suggested to the last author to derive finite twisted honeycombs from 3dimensional hyperbolic tessellations with horospherical facets and/or vertex-figures producing therefore rank 4 objects with toroidal facets and/or vertex-figures. About the same time Grünbaum [23] suggested to study abstract objects, which he called polystroma, whose faces and vertex-figures are not necessarily spherical. Inspired by the ideas of Coxeter and Grünbaum, in 1982 Schulte and Danzer [18] formalised and began developing the theory of regular abstract polytopes (which they named incidence polytopes). In 1984, Colbourn and Weiss [8] unaware of the work of Schulte and Danzer, published a census of regular and chiral finite rank 4 polystroma derived from hyperbolic tessellations by applying the "twisted honeycomb" method of Coxeter. Not all such objects satisfied a more restrictive condition of abstract polytopality of [18].

By late 1980s a number of sporadic examples of chiral abstract polytopes in rank 3 and 4 were found. In 1991 Schulte and the last author of this paper developed the basic structure theory of abstract chiral polytopes of any rank [37] and in particular characterised their automorphism groups. These objects are now quite well understood and have been studied extensively over the past 30 years. Schulte, Monson and Weiss developed various methods of constructing such polytopes in rank 4. However, the classical approach to constructing higher rank polytopes inductively from the lower rank ones proved to be impossible for chiral polytopes. Although in 1995 [39] there was a universal extension method found leading to 
rank 5 chiral polytopes with regular facets, no chiral finite polytopes were known to exist in rank 5 or higher. It was only in 2008 that Conder, Hubard and Pisanski produced the first examples of finite higher rank chiral polytopes [10] and in 2010 Pellicer [36] gave a construction for arbitrary rank.

In [24], Hartley, Hubard and Leemans constructed two atlases of chiral polytopes. Firstly they sought them as quotients of regular polytopes arising from the Atlas of Small Regular Polytopes (http://www.abstract-polytopes.com/atlas/); secondly, for each almost simple group $\Gamma$ such that $S \leq \Gamma \leq A u t(S)$ where $S$ is a simple group of order less than 900,000 listed in the Atlas of Finite Groups, they gave, up to isomorphism, the abstract chiral polytopes on which $\Gamma$ acts regularly. Such an atlas existed already in the regular case [28]. These atlases turned out to be very inspiring to find patterns and get classification results (see $[26,20,27]$ for instance).

An abstract regular or chiral polytope is an incidence geometry with a string diagram. Recently, the authors have defined the notion of hypertope in [21] with the idea to allow more general diagrams than string diagrams. The present paper can be viewed as the beginning of an ambitious project to construct rank 4 hypertopes from their rank 3 residues. Among all hypertopes having some prescribed residues of rank 3, that are either spherical or toroidal maps or hypermaps, we consider whenever possible, the universal one (that is the one covering all hypertopes of this kind). The possible (non-string) diagrams for such hypertopes are listed in Figure 1. In this paper we only consider the first three diagrams (hence the "hexagonal extensions" in the title) leaving other diagrams for future work.

The paper is organised as follows. In Section 2, we give the definitions and notation needed to understand this paper. In Section 3, we explain what are rank four universal locally toroidal hypertopes. In Section 4, we study locally toroidal regular and chiral polytopes of type $\{6,3,6\}$. In Section 5 , we study locally toroidal regular and chiral polytopes of type $\{3,6,3\}$. In Section 6 , we give examples of hexagonal extensions of toroidal hypermaps of type $(3,3,3)$. In Section 7 , we give examples of nonlinear hexagonal extensions of the tetrahedron and, among these examples, a new infinite family of finite regular hypertopes arises. In Section 8, we give examples of 4-circuits with hexagonal residues. Finally, we conclude the paper in Section 9 by stating some conjectures and open problems.

As to notation for groups, we follow the Atlas of Finite Groups [13].

\section{Preliminaries}

2.1. Hypertopes. As in [5], an incidence system $\Gamma:=(X, *, t, I)$ is a 4 -tuple such that

- $X$ is a set whose elements are called the elements of $\Gamma$;

- $I$ is a set whose elements are called the types of $\Gamma$;

- $t: X \rightarrow I$ is a type function, associating to each element $x \in X$ of $\Gamma$ a type $t(x) \in I$

- $*$ is a binary relation on $X$ called incidence, that is reflexive, symmetric and such that for all $x, y \in X$, if $x * y$ and $t(x)=t(y)$ then $x=y$.

The incidence graph of $\Gamma$ is the graph whose vertex set is $X$ and where two vertices are joined provided the corresponding elements of $\Gamma$ are incident. A flag is a set of pairwise incident elements of $\Gamma$, i.e. a clique of its incidence graph. The type of a flag $F$ is $\{t(x): x \in F\}$. A chamber is a flag of type $I$. An element $x$ is incident to a flag $F$, and we write $x * F$ for that, provided $x$ is incident to all elements of 
$F$. An incidence system $\Gamma$ is a geometry or incidence geometry provided that every flag of $\Gamma$ is contained in a chamber (or in other words, every maximal clique of the incidence graph is a chamber). The rank of $\Gamma$ is the number of types of $\Gamma$, namely the cardinality of $I$.

Let $\Gamma:=(X, *, t, I)$ be an incidence geometry and $F$ a flag of $\Gamma$. The residue of $F$ in $\Gamma$ is the incidence system $\Gamma_{F}:=\left(X_{F}, *_{F}, t_{F}, I_{F}\right)$ where

- $X_{F}:=\{x \in X: x * F, x \notin F\}$;

- $I_{F}:=I \backslash t(F)$;

- $t_{F}$ and $*_{F}$ are the restrictions of $t$ and $*$ to $X_{F}$ and $I_{F}$.

An incidence system $\Gamma$ is connected if its incidence graph is connected. It is residually connected when each residue of rank at least two of $\Gamma$ has a connected incidence graph. It is called thin (resp. firm) when every residue of rank one of $\Gamma$ contains exactly two (resp. at least two) elements.

An incidence system $\Gamma:=(X, *, t, I)$ is chamber-connected when for each pair of chambers $C$ and $C^{\prime}$, there exists a sequence of successive chambers $C=: C_{0}$, $C_{1}, \ldots, C_{n}:=C^{\prime}$ such that $\left|C_{i} \cap C_{i+1}\right|=|I|-1$. An incidence system $\Gamma:=(X, *, t, I)$ is strongly chamber-connected when all its residues of rank at least 2 of $\Gamma$ (including $\Gamma$ itself) are chamber-connected.

Proposition 2.1. [21, Proposition 2.1] Let $\Gamma$ be a firm incidence geometry. Then $\Gamma$ is residually connected if and only if $\Gamma$ is strongly chamber-connected.

A hypertope is a thin incidence geometry which is strongly chamber connected or equivalently residually connected.

2.2. Regular and chiral hypertopes as $C^{+}$-groups. Let $\Gamma:=(X, *, t, I)$ be an incidence system. An automorphism of $\Gamma$ is a mapping $\alpha:(X, I) \rightarrow(X, I)$ where

- $\alpha$ is a bijection on $X$;

- for each $x, y \in X, x * y$ if and only if $\alpha(x) * \alpha(y)$;

- for each $x, y \in X, t(x)=t(y)$ if and only if $t(\alpha(x))=t(\alpha(y))$.

An automorphism $\alpha$ of $\Gamma$ is called type preserving when for each $x \in X, t(\alpha(x))=$ $t(x)$ (i.e. $\alpha$ maps each element on an element of the same type).

The set of type-preserving automorphisms of $\Gamma$ is a group denoted by $\operatorname{Aut}_{I}(\Gamma)$. The set of automorphisms of $\Gamma$ is a group denoted by $A u t(\Gamma)$. Elements of $A u t(\Gamma) \backslash$ Aut $_{I}(\Gamma)$ are called correlations.

An incidence system $\Gamma$ is flag-transitive if $A_{u t}(\Gamma)$ is transitive on all flags of a given type $J$ for each type $J \subseteq I$. An incidence system $\Gamma$ is chamber-transitive if $\operatorname{Aut}_{I}(\Gamma)$ is transitive on all chambers of $\Gamma$. Observe that if $\Gamma$ is a firm incidence geometry, flag-transivity and chamber-transitivity are equivalent. Finally, an incidence system $\Gamma$ is regular if $A u t_{I}(\Gamma)$ acts regularly on the chambers (i.e. the action is semi-regular and transitive). A regular hypertope is a flag-transitive hypertope (note that thinness implies that the action of $A u t_{I}(\Gamma)$ is free).

Given an incidence system $\Gamma$ and a chamber $C$ of $\Gamma$, we may associate to the pair $(\Gamma, C)$ the pair consisting of the automorphism group $G:=\operatorname{Aut}_{I}(\Gamma)$ and the set $\left\{G_{i}: i \in I\right\}$ of subgroups of $G$ where $G_{i}$ is the stabiliser in $G$ of the element of type $i$ in $C$.

In the case of a regular hypertope $\Gamma$, the subgroups $\cap_{j \in I \backslash\{i\}} G_{j}$ are cyclic groups of order 2 and we denote their generators $\rho_{i}$ 's. The set $\left\{\rho_{i}: i \in I\right\}$ generates 
$\operatorname{Aut}_{I}(\Gamma)$ (see [19]) and for that reason is called the set of distinguished generators of $\operatorname{Aut}_{I}(\Gamma)$.

The following proposition shows how to start from a group and some of its subgroups and construct an incidence system.

Proposition 2.2. (Tits, 1956) [42] Let $n$ be a positive integer and $I:=\{1, \ldots, n\}$. Let $G$ be a group together with a family of subgroups $\left(G_{i}\right)_{i \in I}, X$ the set consisting of all cosets $G_{i} g, g \in G, i \in I$ and $t: X \rightarrow I$ defined by $t\left(G_{i} g\right)=i$. Define an incidence relation $*$ on $X \times X$ by :

$$
G_{i} g_{1} * G_{j} g_{2} \text { iff } G_{i} g_{1} \cap G_{j} g_{2} \text { is non-empty in } G \text {. }
$$

Then the 4-tuple $\Gamma:=(X, *, t, I)$ is an incidence system having a chamber. Moreover, the group $G$ acts by right multiplication on $\Gamma$ as a group of type preserving automorphisms. Finally, the group $G$ is transitive on the flags of rank less than 3.

When a geometry $\Gamma$ is constructed using the proposition above, we denote it by $\Gamma\left(G ;\left(G_{i}\right)_{i \in I}\right)$.

Consider a pair $\left(G^{+}, R\right)$ with $G^{+}$being a group and $R:=\left\{\alpha_{1}, \ldots, \alpha_{r-1}\right\}$ a set of generators of $G^{+}$. Define $\alpha_{0}:=1_{G^{+}}$and $\alpha_{i j}:=\alpha_{i}^{-1} \alpha_{j}$ for all $0 \leq i, j \leq r-1$. Observe that $\alpha_{j i}=\alpha_{i j}^{-1}$. Let $G_{J}^{+}:=\left\langle\alpha_{i j} \mid i, j \in J\right\rangle$ for $J \subseteq\{0, \ldots, r-1\}$. If the pair $\left(G^{+}, R\right)$ satisfies condition (2.1) below called the intersection condition $\mathrm{IC}^{+}$, we say that $\left(G^{+}, R\right)$ is a $C^{+}$-group.

$$
\forall J, K \subseteq\{0, \ldots, r-1\} \text {, with }|J|,|K| \geq 2, G_{J}^{+} \cap G_{K}^{+}=G_{J \cap K}^{+} .
$$

Two chambers $C$ and $C^{\prime}$ of an incidence geometry of rank $r$ are called $i$-adjacent if $C$ and $C^{\prime}$ differ only in their $i$-elements. When the geometry is thin we denote $C^{\prime}$ by $C^{i}$. Let $\Gamma(X, *, t, I)$ be a thin incidence geometry. We say that $\Gamma$ is chiral if $\operatorname{Aut}_{I}(\Gamma)$ has two orbits on the chambers of $\Gamma$ such that any two adjacent chambers lie in distinct orbits.

Given a chiral hypertope $\Gamma(X, *, t, I)$ (with $I:=\{0, \ldots, r-1\})$ and its automorphism group $G^{+}:=\operatorname{Aut}_{I}(\Gamma)$, pick a chamber $C$. For any pair $i \neq j \in I$, there exists a unique automorphism $\alpha_{i j} \in G^{+}$that maps $C$ to $\left(C^{i}\right)^{j}$. Also, $C \alpha_{i i}=\left(C^{i}\right)^{i}=C$ and $\alpha_{i j}^{-1}=\alpha_{j i}$. We define the distinguished generators of $G^{+}$with respect to a base chamber $C$ as follows:

$$
\alpha_{0}:=1_{G^{+}}, \alpha_{i}:=\alpha_{0 i}(i=1 \ldots, r-1) .
$$

Define $\alpha_{i j}:=\alpha_{i}^{-1} \alpha_{j}$ for all $0 \leq i, j \leq r-1$. Let $G_{J}^{+}:=\left\langle\alpha_{i j} \mid i, j \in J\right\rangle$ for $J \subseteq\{0, \ldots, r-1\}$.

Theorem 2.3. [21, Theorem 7.1] Let $I:=\{0, \ldots, r-1\}$ and let $\Gamma$ be a chiral hypertope of rank $r$. Let $C$ be a chamber of $\Gamma$. The pair $\left(G^{+}, R\right)$, where $G^{+}=$ Aut $_{I}(\Gamma)$ and $R$ is the set of distinguished generators of $\Gamma$ with respect to $C$, is a $C^{+}$-group

Regular and chiral hypertopes can be constructed from some $\mathrm{C}^{+}$-groups. We recall how to construct a coset geometry from a group and an independent generating set of this group.

Construction 2.1. [21, Construction 8.1] Let $G^{+}$be a group and $R:=\left\{\alpha_{1}, \ldots, \alpha_{r-1}\right\}$ be an independent generating set of $G^{+}$. Define $G_{i}:=\left\langle\alpha_{j} \mid j \neq i\right\rangle$ for $i=1, \ldots, r-1$ and $G_{0}:=\left\langle\alpha_{1}^{-1} \alpha_{j} \mid j \geq 2\right\rangle$. The coset geometry $\Gamma\left(G^{+}, R\right):=\Gamma\left(G^{+} ;\left(G_{i}\right)_{i \in\{0, \ldots, r-1\}}\right)$ constructed using Proposition 2.2 is the geometry associated to the pair $\left(G^{+}, R\right)$. 
The coset geometry $\Gamma\left(G^{+}, R\right)$ gives an incidence system using Proposition 2.2. This incidence system is not necessarily a thin geometry, nor is it necessarily residually connected. But if it is, then it is a hypertope and if its automorphism group has at most two orbits on its flags, the following theorem gives a way to check whether this geometry is chiral or regular.

Theorem 2.4. [21, Theorem 8.2] Let $\left(G^{+}, R\right)$ be a $C^{+}$-group. Let $\Gamma:=\Gamma\left(G^{+}, R\right)$ be the coset geometry associated to $\left(G^{+}, R\right)$ using Construction 2.1. If $\Gamma$ is a hypertope and $G^{+}$has two orbits on the set of chambers of $\Gamma$, then $\Gamma$ is chiral if and only if there is no automorphism of $G^{+}$that inverts all the elements of $R$. Otherwise, there exists an automorphism $\sigma \in A u t\left(G^{+}\right)$that inverts all the elements of $R$ and the group $G^{+}$extended by $\sigma$ is regular on $\Gamma$.

Later in the paper, when we will build hypertopes from their $C^{+}$-groups given as finitely presented groups, we will indeed check that the corresponding incidence system is thin and residually connected. This check is most of the time easily performed with MaGma. We will list the hypertopes obtained in tables, not mentioning those presentations giving a $C^{+}$-group that does not yield a hypertope.

2.3. B-diagrams. Let $R:=\left\{\alpha_{1}, \ldots, \alpha_{r-1}\right\}$ and $G^{+}=\langle R\rangle$ be such that $\left(G^{+}, R\right)$ is a $\mathrm{C}^{+}$-group. It is convenient to represent $\left(G^{+}, R\right)$ by the following complete graph with $r$ vertices which we will call the $B$-diagram (short for Buekenhout) of $\left(G^{+}, R\right)$ and denote by $\mathcal{B}\left(G^{+}, R\right)$. The vertex set of $\mathcal{B}$ is the set $\left\{\alpha_{0}, \ldots, \alpha_{r-1}\right\}$. The edges $\left\{\alpha_{i}, \alpha_{j}\right\}$ of this graph are labelled by $o\left(\alpha_{i}^{-1} \alpha_{j}\right)=o\left(\alpha_{j}^{-1} \alpha_{i}\right)=o\left(\alpha_{i} \alpha_{j}^{-1}\right)$. We take the convention of dropping an edge if its label is 2 and of not writing the label if it is 3 . Vertices of $\mathcal{B}$ are represented by small circles in order to distinguish from the vertices of a Coxeter diagram, which represent involutions. A regular or chiral polytope can be defined as a regular or chiral hypertope with linear Coxeter diagram, or equivalently, with linear B-diagram.

Rank four extensions of rank three toroidal polytopes of type $\{6,3\}_{(a, b)}$ have been studied by Schulte and Weiss [38], Nostrand and Schulte [33] and Monson and Weiss [32]. The rotation subgroup of the automorphism group of a rank three toroidal polytope $\mathcal{P}:=\{6,3\}_{(a, b)}$ is the group $G^{+}:=\operatorname{Aut}(\mathcal{P})^{+}$defined as follows.

$$
G^{+}:=\left\langle x, y \mid x^{6}, y^{3},\left(x^{-1} y\right)^{2},\left(y^{-1} x^{-2}\right)^{a}\left(y x^{2}\right)^{b}\right\rangle .
$$

The B-diagram of $\left(G^{+},\{x, y\}\right)$ is the following.

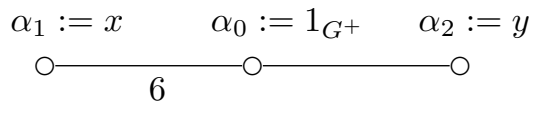

Recall that the polytope above is obtained by identifying opposite sides of a parallelogram in the tessellation of the Euclidean plane by hexagons to obtain the $\operatorname{map}\{6,3\}_{(a, b)}$.

The dual of $\mathcal{P}$ will be the polytope $\mathcal{P}^{*}:=\{3,6\}_{(a, b)}$ with rotation group $H^{+}:=$ $\operatorname{Aut}\left(\mathcal{P}^{*}\right)^{+}$defined as follows.

$$
H^{+}:=\left\langle x, y \mid x^{3}, y^{6},\left(x^{-1} y\right)^{2},\left(x^{-1} y^{-2}\right)^{a}\left(x y^{2}\right)^{b}\right\rangle .
$$

Observe that presentation (2.4) is obtained by interchanging $x$ and $y$ in presentation (2.3). The B-diagram of $\left(H^{+},\{x, y\}\right)$ is the following. 


$$
\alpha_{1}:=x \quad \alpha_{0}:=1_{H^{+}} \quad \alpha_{2}:=y
$$

Indeed there is no distinction between the $\mathrm{C}^{+}$-groups of a polytope and its dual. We make the distinction when we write the B-diagram (ranking the generators).

The rotation subgroup of the automorphism group of a rank three toroidal polytope $\mathcal{P}:=\{4,4\}_{(a, b)}$ is the group $G^{+}:=\operatorname{Aut}(\mathcal{P})^{+}$defined as follows.

$$
G^{+}:=\left\langle x, y \mid x^{4}, y^{4},\left(x^{-1} y\right)^{2},(x y)^{a}\left(x^{-1} y^{-1}\right)^{b}\right\rangle \text {. }
$$

The B-diagram of $\left(G^{+},\{x, y\}\right)$ is the following.

$$
\alpha_{1}:=x \quad \alpha_{0}:=1_{G^{+}} \quad \alpha_{2}:=y
$$

Observe that the dual $\mathcal{P}^{*}$ of $\mathcal{P}$ is $\{4,4\}_{(a,-b)}=\{4,4\}_{(b, a)}$ as the vectors $(a,-b)$ and $(b, a)$ are orthogonal (the characterisation of dual polytopes it terms of the rotational groups can be found for instance in [37]). The rotational group for $\mathcal{P}^{*}$ is obtained by interchanging $x$ with $y$.

The rotation subgroup of the automorphism group of a rank three toroidal hypermap $\mathcal{P}:=(3,3,3)_{(a, b)}$ is the group $G^{+}:=\operatorname{Aut}(\mathcal{P})^{+}$defined as follows.

$$
G^{+}:=\left\langle x, y \mid x^{3}, y^{3},\left(x^{-1} y\right)^{3},\left(x y^{-1} x\right)^{a}(x y)^{b}\right\rangle .
$$

The B-diagram of $\left(G^{+},\{x, y\}\right)$ is the following.

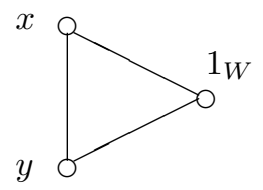

The elements corresponding to the three possible types in a hypermap are called hypervertices, hyperedges and hyperfaces. The dual of a hypermap is obtained interchanging hypervertices with hyperfaces. The dual $\mathcal{P}^{*}$ of $\mathcal{P}$ is $(3,3,3)_{(b, a)}$.

\section{RANK 4 UNIVERSAL LOCALLY TOROIDAL HYPERTOPES}

Spherical hypertopes (in the sense of Coxeter) of rank 3 are maps (polyhedra) on the sphere while toroidal hypertopes of rank 3 are either maps or hypermaps on the torus. The toroidal (regular or chiral) hypertopes of rank 3 are divided into the following families: the toroidal maps $\{3,6\}_{(b, c)},\{6,3\}_{(b, c)},\{4,4\}_{(b, c)}$, and the hypermaps $(3,3,3)_{(b, c)}$ with $(b, c) \neq(1,1)$. Note that the hypermap $(3,3,3)_{(b, c)}$ is obtained from the toroidal map $\{6,3\}_{(b, c)}$ by doubling the fundamental region. Indeed as $\{6,3\}_{(b, c)}$ is bipartite it is possible to take one monochromatic set of vertices to be the hyperedges of the hypermap $(3,3,3)_{(b, c)}$ (see [44]). But in the case $(b, c)=(1,1)$ the corresponding incidence graph is a complete tripartite graph and, therefore, the geometry is not thin (see [21]). Indeed that is the unique highly symmetric (regular or chiral) toroidal hypermap that is not an hypertope.

Similarly to the theory of abstract regular polytopes it is possible to construct hypertopes inductively from hypertopes of lower rank. In the case of polytopes $\left\{P_{1}, P_{2}\right\}$ denotes a polytope having facets isomorphic to $P_{1}$ and vertex-figures isomorphic to $P_{2}$ (see [30]). More precisely, if the set of regular polytopes having facets 


\begin{tabular}{|c|c|c|c|c|}
\hline$p$ & $\mathbf{s}$ & $g$ & Group & Chiral/Regular \\
\hline \multirow[t]{8}{*}{3} & $(2,0)$ & 240 & $S_{5} \times C_{2}$ & regular \\
\hline & $(3,0)$ & 1296 & {$\left[\begin{array}{lll}1 & 1 & 2\end{array}\right]^{3} \rtimes C_{2}$} & regular \\
\hline & $(4,0)$ & 15360 & {$\left[\begin{array}{lll}1 & 1 & 2\end{array}\right]^{4} \rtimes C_{2}$} & regular \\
\hline & $(1,2)$ & 336 & $P G L_{2}(7)$ & chiral \\
\hline & $(1,3)$ & 2184 & $L_{2}(13) \times C_{2}$ & chiral \\
\hline & $(1,4)$ & 8064 & $S L_{2}(7) \rtimes A_{4} \rtimes C_{2}$ & chiral \\
\hline & $(2,2)$ & 2880 & $S_{5} \times S_{4}$ & regular \\
\hline & $(2,3)$ & 6840 & $P G L_{2}(19)$ & chiral \\
\hline \multirow[t]{3}{*}{4} & $(1,1)$ & 288 & $S_{3} \rtimes[3,4]$ & regular \\
\hline & $(2,0)$ & 768 & {$[3,3,4] \times C_{2}$} & regular \\
\hline & $(1,2)$ & 2016 & $P G L_{2}(7) \times S_{3}$ & chiral \\
\hline 5 & $(2,0)$ & 28800 & {$[3,3,5] \times C_{2}$} & regular \\
\hline
\end{tabular}

TABLE 1. Known finite polytopes of type $\left\{\{6,3\}_{\mathbf{s}},\{3, p\}\right\}$ with $p \in\{3,4,5\}$ (having $g$ flags)

$P_{1}$ and vertex-figures $P_{2}$, denoted by $\left\langle P_{1}, P_{2}\right\rangle$, is nonempty, there exists a regular polytope that covers every other element of the set $\left\langle P_{1}, P_{2}\right\rangle$, that is the universal regular polytope $\left\{P_{1}, P_{2}\right\}$. In addition if the automorphism group of the universal polytope $\left\{P_{1}, P_{2}\right\}$ is the group $\left\langle\rho_{0}, \ldots, \rho_{n}\right\rangle$, the automorphism groups of $P_{1}$ and $P_{2}$ are $\left\langle\rho_{0}, \ldots, \rho_{n-1}\right\rangle$ and $\left\langle\rho_{1}, \ldots, \rho_{n}\right\rangle$, respectively.

In a similar way here, we construct rank 4 regular and chiral hypertopes that we call universal when the relations corresponding to each rank 3 residue of the resulting hypertope together with the relations implicit in the B-diagram of the hypertope determine the group.

Here we consider universal locally toroidal hypertopes of rank 4, meaning that all residues of rank 3 are either spherical or toroidal, with at least one being toroidal. These hypertopes are finite whenever their automorphism group is finite.

The existence of regular universal locally toroidal polytopes of rank 4 is investigated in [30], moreover the authors give an enumeration of finite locally toroidal universal polytopes. For the universal polytopes $\left\{\{4,4\}_{(b, c)},\{4,4\}_{(e, f)}\right\}$ a nearly complete finiteness characterisation is given, for $\left\{\{4,4\}_{(b, c)},\{4,3\}\right\},\left\{\{6,3\}_{(b, c)},\{3, p\}\right\}$ with $p \in\{3,4,5\}$ and $\left\{\{6,3\}_{(b, c)},\{3,6\}_{(e, f)}\right\}$ the enumeration is complete, and for the polytopes $\left\{\{3,6\}_{(b, c)},\{6,3\}_{(e, f)}\right\}$ partial results are known.

In [21] we also list the known finite universal chiral polytopes $\left\{\{6,3\}_{(b, c)},\{3, p\}\right\}$ for $p \in\{3,4,5\}$ and we conjecture that the list is complete. We give this list in Table 1.

In Figure 1, we list the diagrams of all possible finite universal locally toroidal hypertopes of rank 4 having nonlinear diagram (where $p \in\{3,4,5,6\}$ ).

The finite universal locally toroidal hypertopes with diagram (1), when $p \neq 6$, have only one toroidal residue that is the hypermap $(3,3,3)_{(b, c)}$, all the remaining residues are spherical. We denote this hypertope by $(3,3,3 ; p)_{(b, c)}$.

In [21] we proved that when $p \in\{3,4,5\}$ and $(b, c) \neq(1,1)$, the regular hypertope $(3,3,3 ; p)_{(b, c)}$ exists (is finite) if and only if the universal regular polytope $\left\{\{6,3\}_{(b, c)},\{3, p\}\right\}$ exists (is finite).

In section 6 we consider the diagram (1) with $p=6$, that we call hexagonal extension of the toroidal hypermaps $(3,3,3)_{(b, c)}$. In this case there are three toroidal 
(1)<smiles>Op1oo1</smiles>

(6)
(2)

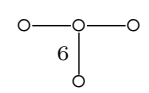

(7)
(3)

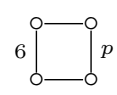

(8)

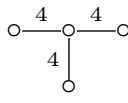

(4)

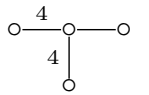

(9)

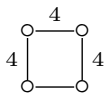

(5)

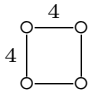

(10)

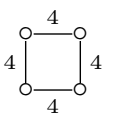

Figure 1. Possible nonlinear diagrams of rank 4 universal locally toroidal hypertopes, where $p \in\{3,4,5,6\}$

residues, that explains why the case $p=6$ is substantially more complex than the cases $p \in\{3,4,5\}$ studied in [21].

In section 7 we deal with the finite universal polytopes with diagram (2) and in section 8 with the universal hypertopes of diagram (3). The universal locally toroidal regular hypertopes with diagram (4) can be obtaining from the regular universal locally toroidal polytope of type $\{4,4,3\}$. Indeed each of them admits a correlation $\tau$ as shown in the following figure.

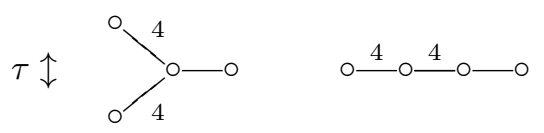

In the case that the toroidal residue is the map $\{4,4\}_{(s, 0)}$ satisfying a relation $\left(\rho_{0} \rho_{1} \rho_{2} \rho_{1}\right)^{s}=1$ (the central vertex of the B-diagram being $\rho_{1}$ ), adding the automorphism $\tau$, such that $\rho_{2}^{\tau}=\rho_{0}$ (and fixing the remaining generators), we get the automorphism group of $\left\{\{4,4\}_{(s, s)},\{4,3\}\right\}$ (generated by $\rho_{0}, \rho_{1}$ and $\tau$ ). Indeed apart from the relations corresponding to the type $\{4,4,3\}$ we get $\left(\rho_{0} \rho_{1} \rho_{0}^{\tau} \rho_{1}\right)^{s}=1$. If the toroidal residue is $\{4,4\}_{(s, s)}$ satisfying a relation $\left(\rho_{0} \rho_{1} \rho_{2}\right)^{2 s}=1$ then adding the automorphism $\tau$ we obtain the group of $\left\{\{4,4\}_{(2 s, 0)},\{4,3\}\right\}$. Thus all regular universal locally toroidal hypertopes of this type are determined by the correspondent universal locally toroidal regular polytope of type $\{4,4,3\}$.

The universal locally toroidal regular hypertopes with diagram (5) also admit a correlation.

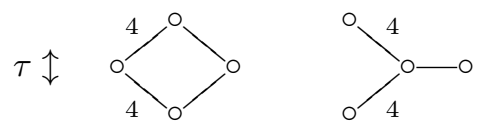

Computer experiments suggest that using this correlation, we get hypertopes. The hypertopes with this diagram and toroidal residue $\{4,4\}_{(s, 0)}$ can be derived from the universal locally toroidal hypertopes with diagram (4) and toroidal residue $\{4,4\}_{(s, s)}$ (indeed if $\rho_{1}$ corresponds to the middle vertex of the toroidal residue, $\left(\rho_{0} \rho_{1} \rho_{2} \rho_{1}\right)^{s}=1, \rho_{0}^{\tau}=\rho_{2}$ and $\rho_{1}^{\tau}=\rho_{1}$, conjugating $\left(\rho_{0} \rho_{1} \rho_{2} \rho_{1}\right)^{s}=1$ by $\tau$ we get $\left.\left(\rho_{2} \rho_{1} \tau\right)^{2 s}=1\right)$. The hypertopes with diagram (5) and toroidal residue $\{4,4\}_{(s, s)}$ can be derived from the universal locally toroidal hypertopes with diagram (4) and 
toroidal residue $\{4,4\}_{(2 s, 0)}$. Again the regular universal locally toroidal hypertopes of these types are determined.

The universal locally toroidal regular hypertopes with diagram being a tetrahedron, as in (6), have four toroidal residues (hypermaps of type $(3,3,3)$ ) corresponding to the four faces of the tetrahedron. The case with all toroidal residues being regular hypermaps of type $(3,3,3)$ is completely studied in [30], where this diagrams are denoted by $\mathcal{T}_{4}\left(q_{1}, q_{2}, q_{3}, q_{4}\right)$ with reflexion group $G\left(q_{1}, q_{2}, q_{3}, q_{4}\right)$. The results are summarised in the following theorems.

Theorem 3.1. [30, Theorem 9E14] $G(s, s, q, q)$ is finite if and only if $s=2$ and $q \in\{2,3,4\}$ (up to an interchange of $s$ and $q$ ).

Theorem 3.2. [30, Theorem 9E15] $G\left(q_{1}, q_{2}, q_{3}, q_{4}\right)$ is infinite in at least the following cases:

(1) $p \mid q_{1}, \ldots, q_{4}$ for some $p \geq 3$;

(2) $p \mid q_{1}$ and $3 p \mid q_{2}, q_{3}, q_{4}$ for some $p \geq 2$.

Theorem 3.3. [30, Theorem 9E17] The group $G\left(q_{1}, q_{2}, q_{3}, q_{4}\right)$ with $\left(q_{1}, q_{2}\right) \in$ $\left\{\left(q_{1}, 3\right),\left(3, q_{2}\right),(4,4),(4,5),(5,4)\right\}$ and $\left(q_{3}, q_{4}\right) \in\left\{\left(q_{3}, 3\right),\left(3, q_{4}\right),(4,4),(4,5),(5,4)\right\}$ is infinite except when $\left(q_{1}, q_{2}, q_{3}, q_{4}\right)=\left(q_{1}, 3,3,2\right)$ (up to an interchange of the pairs $\left(q_{1}, q_{2}\right)$ and $\left.\left(q_{3}, q_{4}\right)\right)$.

The remaining possibilities for a universal locally toroidal hypertope with diagram being a tetrahedron will be studied in future work, as well as the locally toroidal polytopes with diagrams (7), (8), (9) and (10).

\section{Locally toroidal REgUlar AND ChiRAL POLYTOPES OF TYPE $\{6,3,6\}$}

Branko Grünbaum first posed the question of classifying regular locally toroidal polystromas in 1977 (see [23]). About the same time Coxeter and Shephard independently constructed in [17] such an object. Several attempts have been made at the classification, including Colbourn and Weiss who produced a computergenerated list of regular and chiral examples of all possible types (see [8]). In 2002 McMullen and Schulte in [31] succeeded in classifying all finite regular locally toroidal universal polytopes with Schläfli symbol $\{6,3, p\}$ for $p=3,4,5$ and 6 (see also chapter 11 of [30]). In [21], we present their classification and in addition provide a list of chiral polytopes with $p \leq 5$, for which we conjecture to be complete. Each such regular polytope is associated with a honeycomb of the hyperbolic 3space. Since the horosphere is isomorphic to the euclidean plane, one can tesselate it by regular hexagons, three meeting at a vertex, to obtain a regular polytope embedded in a horosphere. The vertex figures of $\{6,3, p\}$ for $p=3,4,5$ are spherical polyhedra isomorphic to the tetrahedron, octahedron and icosahedron respectively. The size of each of these polyhedra is determined by the dihedral angle which has to be $\frac{2 \pi}{p}$ in order that $p$ facets fit around an edge without overlap. The facets of the hyperbolic honeycombs are therefore horospherical honeycombs $\{6,3\}$ centered at the absolute. All the vertices of the honeycomb are (finite) points of the hyperbolic space. When $p=6$, the vertices of the honeycomb $\{6,3,6\}$ also belong to the absolute. Hence there are no vertices of this honeycomb that belong to the hyperbolic space (but all the edges are there).

McMullen and Schulte used twisting operations on quotients of certain Coxeter groups that are associated with complex hermitian forms. Their results [30, Chapter 


\begin{tabular}{||c|c|c|c||}
\hline $\mathbf{s}$ & $\mathbf{t}$ & $g$ & Group \\
$(2,0)$ & $(2,0)$ & 480 & $S_{5} \times 2^{2}$ \\
$(2,0)$ & $(3,0)$ & 2592 & $D_{12} \times S_{3} \times S_{3}: S_{3}$ \\
$(2,0)$ & $(4,0)$ & 30720 & $2^{1+6}:\left(S_{5} \times 2\right)$ \\
$(2,0)$ & $(2,2)$ & 5760 & $S_{5} \times 2^{3}: S_{3}$ \\
$(s, 0)$ with $s \equiv 0 \bmod 3$ & $(1,1)$ & $72 s^{2}$ & $S_{3}:[3,6]_{(s, 0)}$ \\
$(s, s)$ with $s \geq 1$ & $(1,1)$ & $216 s^{2}$ & $S_{3}:[3,6]_{(s, s)}$ \\
\hline
\end{tabular}

TABLE 2. Finite regular polytopes of type $\left\{\{6,3\}_{\mathbf{s}},\{3,6\}_{\mathbf{t}}\right\}$

\begin{tabular}{||c|c|c|c||}
\hline $\mathbf{s}$ & $\mathbf{t}$ & $g$ & Group \\
\hline$(1,2)$ & $(1,2)$ & 1344 & $2 \cdot L_{2}(7): 2: 2$ \\
$(1,2)$ & $(2,1)$ & 20160 & $2 \cdot A_{7}: 2: 2$ \\
\hline$(2,0)$ & $(1,2)$ & 672 & $L_{2}(7): 2 \times 2$ \\
$(2,0)$ & $(1,3)$ & 4368 & $L_{2}(13) \times 2 \times 2$ \\
$(2,0)$ & $(1,4)$ & 16128 & $Q_{8} \cdot\left(L_{2}(7) \times 2\right): S_{3}$ \\
$(2,0)$ & $(2,3)$ & 13680 & $L_{2}(19): 2 \times 2$ \\
\hline
\end{tabular}

TABLE 3. Known finite chiral polytopes of type $\left\{\{6,3\}_{\mathbf{s}},\{3,6\}_{\mathbf{t}}\right\}$

11] on existence of finite universal polytopes of type $\{6,3,6\}$ are summarised in Table 2.

Using MAGMA, we construct several new finite universal chiral polytopes of this type and we present our results in Table 3. In fact, only the first two polytopes appearing in this table were previously known. We conjecture this list to be complete.

Consider the Coxeter diagram with usual generators $\rho_{i}(i=0, \ldots, 3)$ as follows.

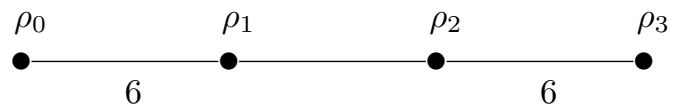

It gives the Coxeter group $W:=[6,3,6]$. Its rotational subgroup $W^{+}=\left\langle\alpha_{1}, \alpha_{2}, \alpha_{3}\right\rangle$ with distinguished generators

$$
\alpha_{0}=1_{W} ; \alpha_{1}=\rho_{1} \rho_{0} ; \alpha_{2}=\rho_{1} \rho_{2} ; \alpha_{3}=\rho_{1} \rho_{3} .
$$

is a $\mathrm{C}^{+}$-group. We now write the $\mathrm{B}$-diagram associated to $\left(W^{+},\left\{\alpha_{1}, \alpha_{2}, \alpha_{3}\right\}\right)$ as follows.

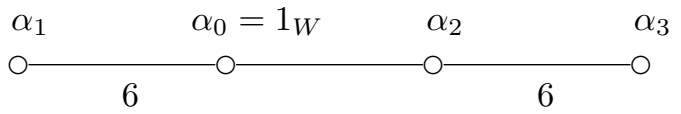

Given the base flag $C$ of the universal polytope $\{6,3,6\}$ such that $\rho_{i}(C)=C^{i}$, $(i=0, \ldots, 3)$, it follows that $\alpha_{1}(C)=\left(C^{1}\right)^{0}, \alpha_{2}(C)=\left(C^{1}\right)^{2}$ and $\alpha_{3}(C)=\left(C^{1}\right)^{3}$.

The automorphism groups of the polytopes of Tables 2 and 3 are obtained using the following presentation where $x:=\alpha_{1}, y:=\alpha_{2}, z:=\alpha_{3}, \mathbf{s}=(a, b)$ and $\mathbf{t}=(c, d)$.

$G^{+}:=\left\langle x, y, z \mid x^{6}, y^{3}, z^{2},\left(x^{-1} y\right)^{2},\left(y^{-1} z\right)^{6},(z x)^{2},\left(y^{-1} x^{-2}\right)^{a}\left(y x^{2}\right)^{b},\left(y(z y)^{2}\right)^{c}\left(y z y^{-1} z\right)^{d}\right\rangle$

Note that, when the polytope is regular, the presentation above gives the rotational subgroup of the full automorphism group. 


\begin{tabular}{||c|c|c|c|c||}
\hline $\mathbf{s}$ & $\mathbf{t}$ & $g$ & Group & \\
\hline$(2,0)$ & $(2,0)$ & 240 & $A_{5}: 2 \times 2$ & regular \\
$(2,0)$ & $(6,0)$ & 720 & $S_{5} \times S_{3}$ & regular \\
$(3,0)$ & $(3,0)$ & 2916 & $3^{1+2} \times 3: S_{3}: S_{3}$ & regular \\
$(3,0)$ & $(4,0)$ & 241920 & $A u t\left(L_{3}(4)\right)$ & regular \\
$(3,0)$ & $(1,1)$ & 324 & $3^{1+2}: 2: S_{3}$ & regular \\
$(3,0)$ & $(2,2)$ & 41472 & $2^{1+6}: 3^{2}: S_{3}: S_{3}$ & regular \\
$(1,1)$ & $(1,1)$ & 108 & $3^{1+2}: 2^{2}$ & regular \\
$(2,2)$ & $(2,2)$ & 13271040 & $2^{5+6}: A_{5} \times 3: S_{3}: S_{3}$ & regular \\
\hline$(3,0)$ & $(1,3)$ & 33696 & $L_{3}(3): S_{3}$ & chiral \\
$(4,0)$ & $(1,2)$ & 12096 & $U_{3}(3): 2$ & chiral \\
$(6,0)$ & $(1,2)$ & 756000 & $U_{3}(5): S_{3}$ & chiral \\
$(1,2)$ & $(3,6)$ & 2016 & $2 \cdot L_{2}(7): S_{3}$ & chiral \\
$(1,2)$ & $(4,4)$ & 36288 & $U_{3}(3): S_{3}$ & chiral \\
$(1,2)$ & $(6,6)$ & 2268000 & $3 \cdot U_{3}(5): S_{3}$ & chiral \\
$(3,5)$ & $(2,1)$ & 672 & $2 \cdot L_{2}(7): 2$ & chiral \\
$(1,4)$ & $(2,1)$ & 2016 & $2 \cdot L_{2}(7): S_{3}$ & chiral \\
$(1,2)$ & $(1,2)$ & 672 & $2 \cdot L_{2}(7): 2$ & chiral \\
\hline
\end{tabular}

TABLE 4. Known finite regular and chiral universal polytopes of type $\left\{\{3,6\}_{\mathbf{s}},\{6,3\}_{\mathbf{t}}\right\}$

Extending the methods that were developed in [21], in Section 6 we will look at hypertopes with a nonlinear diagram, arising from these groups.

\section{Polytopes of type $\{3,6,3\}$}

As for the polytopes of the previous section, the polytopes of type $\{3,6,3\}$ have also been studied is several articles and in [30]. We refer to [30, Section 11E] for more details. We now consider the polytopes of type $\left\{\{3,6\}_{(a, b)},\{6,3\}_{(c, d)}\right\}$. We use the following B-diagram, where $x, y$ and $y^{-1} z$ are rotations generating the infinite Coxeter group $[3,6,3]$.

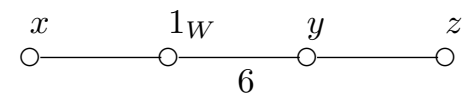

The type $\{3,6\}$ residues, given by the facets and vertex-figures, may be nonisomorphic. Thus we need four parameters $a, b, c, d$ giving two additional relations in the following presentation for the rotation subgroup of the automorphism group $G$. The automorphism groups of the polytopes of Table 4 are obtained using the following presentation where $\mathbf{s}=(a, b)$ and $\mathbf{t}=(c, d)$.

$$
\begin{gathered}
G^{+}(a, b, c, d):=\langle x, y, z| x^{3}, y^{6}, z^{2},\left(x^{-1} z\right)^{2},\left(y^{-1} z\right)^{3},\left(x^{-1} y\right)^{2}, \\
\left.\left(x^{-1} y^{-2}\right)^{a}\left(x y^{2}\right)^{b},\left(z y^{3}\right)^{c}\left(y^{-1} z y^{-2}\right)^{d}\right\rangle
\end{gathered}
$$

We found several new universal hypertopes compared to Table 1 of [8]. In the regular case, lines 5 and 7 are not in [8], but they can be found in Table 11E1 of [30], line 8 of Table 4 is new. In the chiral case, all but the last two are new. 


\section{Hexagonal extensions of toroidal hypermap $(3,3,3)$}

In this section, as we did in [21], starting with the Coxeter group $[6,3,6]$ with diagram

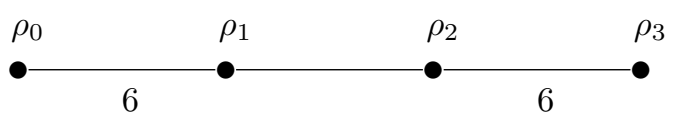

we double the fundamental region so that the resulting involutory generators give us the following Coxeter group that is of index two in $[6,3,6]$.

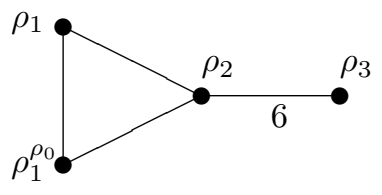

In the geometry constructed from this group, all rank three residues with connected diagrams are either Euclidean tessellations of horospheres of type $\{3,6\}$ (up to duality) or hypermaps of type $(3,3,3)$. We denote by $[(3,3,3), 6]$ the Coxeter group having this diagram.

In order to construct a finite hypertope $\mathcal{H}$ whose residues could be chiral, we consider the rotation subgroup $W^{+}:=[(3,3,3), 6]^{+}$of the group $W:=[(3,3,3), 6]$. The B-diagram of $W^{+}$is as follows, where $x, y$ and $z$ are rotations generating this infinite group.

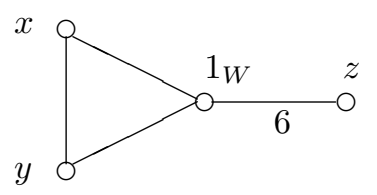

In $\mathcal{H}$ the two type $\{3,6\}$ residues may be nonisomorphic, and in that case they cannot be obtained from the polytopes of type $\left\{\{6,3\}_{s},\{3,6\}_{t}\right\}$ as described before. To describe all regular and chiral hypertopes with the B-diagram above we need six parameters $a, b, c, d, e, f$ giving three additional relations in the following presentation for the rotation subgroup of $G:=\operatorname{Aut}(\mathcal{H})$.

$$
\begin{gathered}
G^{+}(a, b, c, d, e, f):=\langle x, y, z| x^{3}, y^{3}, z^{6},\left(x^{-1} z\right)^{2},\left(y^{-1} z\right)^{2},\left(x^{-1} y\right)^{3}, \\
\left.\left(y x^{-1} y\right)^{a}(y x)^{b},\left(y^{-1} z^{-2}\right)^{c}\left(y z^{2}\right)^{d},\left(x^{-1} z^{-2}\right)^{e}\left(x z^{2}\right)^{f}\right\rangle
\end{gathered}
$$

where the subgroup $\langle x, z\rangle$ acts on a polytope of type $\{3,6\}_{(e, f)},\langle y, z\rangle$ acts on a polytope of type $\{3,6\}_{(c, d)}$, and $\langle x, y\rangle$ acts of a hypermap of type $(3,3,3)_{(a, b)}$. We say that $\mathcal{H}$ has type $\left\{(3,3,3)_{s}, 6\right\}$ where $s=(a, b)$. If there exists a correlation $\delta$ of order two fixing $z$ and interchanging $x$ and $y$, the residues $\{3,6\}_{(e, f)}$ and $\{3,6\}_{(c, d)}$ are isomorphic and therefore $(c, d)=(e, f)$. The automorphism groups of the hypertopes of Table 5 are obtained using the presentation above.

\section{NONLINEAR HEXAGONAL EXTENSIONS OF THE TETRAHEDRON}

We now consider a hypertope $\mathcal{H}$ with the following B-diagram, where $x, y$ and $z$ are rotations generating the corresponding $\mathrm{C}^{+}$-group. 


\begin{tabular}{||c|c|c|c|c|c||}
\hline$(a, b)$ & $(c, d)$ & $(e, f)$ & $g$ & $G$ & Regular/Chiral \\
\hline$(2,0)$ & $(2,0)$ & $(2,0)$ & 240 & $S_{5} \times 2$ & regular \\
$(2,0)$ & $(3,0)$ & $(3,0)$ & 1296 & $S_{3} \times S_{3} \times S_{3}: S_{3}$ & regular \\
$(2,0)$ & $(4,0)$ & $(4,0)$ & 15360 & $2^{1+6}:\left(A_{5}: 2\right)$ & regular \\
$(2,0)$ & $(6,0)$ & $(2,2)$ & 2880 & $S_{5} \times 2^{2}: S_{3}$ & regular \\
$(3,0)$ & $(2,0)$ & $(2,0)$ & 1296 & $6: S_{3}: S_{3}: S_{3}$ & regular \\
$(3,0)$ & $(2,0)$ & $(3,0)$ & 13824 & $2^{1+6}: 3: S_{3}: S_{3}$ & regular \\
$(3,0)$ & $(2,0)$ & $(4,0)$ & 165888 & $2^{1+6}: 6: S_{3}: S_{3}: S_{3}$ & regular \\
$(3,0)$ & $(2,0)$ & $(5,0)$ & 2592000 & $2 \cdot\left(A_{5} \times\left(A_{5} \times A_{5}\right)\right): 3: 2$ & regular \\
$(3,0)$ & $(2,0)$ & $(2,2)$ & 3888 & $S_{3} \times 3: S_{3}: S_{3}: S_{3}$ & regular \\
$(3,0)$ & $(2,0)$ & $(4,4)$ & 248832 & $2^{1+6}:\left(3: S_{3}: S_{3}: 3\right): S_{3}$ & regular \\
$(3,0)$ & $(3,0)$ & $(1,1)$ & 972 & $3^{1+2}: 6: S_{3}$ & regular \\
$(3,0)$ & $(1,1)$ & $(1,1)$ & 324 & $3^{2} \times S_{3}: S_{3}$ & regular \\
$(3,0)$ & $(1,1)$ & $(3,3)$ & 2916 & $3^{1+2} \times 3: S_{3}: S_{3}$ & regular \\
$(3,0)$ & $(2,2)$ & $(2,0)$ & 3888 & $S_{3} \times 3: S_{3}: S_{3}: S_{3}$ & regular \\
$(4,0)$ & $(2,0)$ & $(2,0)$ & 15360 & $4 \cdot\left(2^{4}: A_{5}\right): 2 \times 2$ & regular \\
\hline$(2,0)$ & $(1,2)$ & $(2,1)$ & 336 & $L_{2}(7): 2$ & chiral \\
$(2,0)$ & $(1,3)$ & $(2,5)$ & 2184 & $L_{2}(13) \times 2$ & chiral \\
$(2,0)$ & $(1,4)$ & $(1,2)$ & 336 & $L_{2}(7): 2$ & chiral \\
$(2,0)$ & $(1,4)$ & $(3,6)$ & 8064 & $Q_{8} \cdot L_{2}(7): S_{3}$ & chiral \\
$(2,0)$ & $(2,3)$ & $(3,2)$ & 6840 & $L_{2}(19): 2$ & chiral \\
$(3,0)$ & $(1,2)$ & $(1,2)$ & 275562 & $3^{2+6}: 7: 3: 2$ & chiral \\
\hline
\end{tabular}

TABLE 5. Known finite universal hypertopes of type $\left\{(3,3,3)_{s}, 6\right\}$

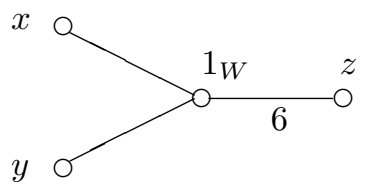

In $\mathcal{H}$ the two type $\{3,6\}$ residues may be nonisomorphic. Thus we need four parameters $a, b, c, d$ giving two additional relations in the following presentation for the the rotation subgroup of $G:=\operatorname{Aut}(\mathcal{H})$.

$$
\begin{gathered}
G^{+}(a, b, c, d):=\langle x, y, z| x^{3}, y^{3}, z^{6},\left(x^{-1} z\right)^{2},\left(y^{-1} z\right)^{2},\left(x^{-1} y\right)^{2}, \\
\left.\left(y^{-1} z^{-2}\right)^{a}\left(y z^{2}\right)^{b},\left(x^{-1} z^{-2}\right)^{c}\left(x z^{2}\right)^{d}\right\rangle .
\end{gathered}
$$

The automorphism groups of the hypertopes of Table 6 are obtained using this presentation where $\mathbf{s}=(a, b)$ and $\mathbf{t}=(c, d)$.

There is an infinite family of regular locally toroidal hypertopes with the following Coxeter diagram having toroidal rank 3 residues $\{3,6\}_{(2,0)}$ and $\{3,6\}_{(s, 0)}$ with $s \geq 3$.

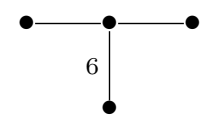

This family of hypertopes can be obtained from the cubic toroids $\{4,3,4\}_{(s, s, 0)}$ whose automorphism group $G$ is the Coxeter group $[4,3,4]=\left\langle\rho_{0}, \rho_{1}, \rho_{2}, \rho_{3}\right\rangle$ factorized by the single extra relation $\left(\rho_{0} \rho_{1} \rho_{2} \rho_{3} \rho_{2}\right)^{2 s}$ (see p.168 of [30]). First consider the hypertope that is obtain from $\{4,3,4\}_{(s, s, 0)}$ using the Petrie operation defined 


\begin{tabular}{|c|c|c|c|c|}
\hline $\mathbf{s}$ & $\mathbf{t}$ & $g$ & $G$ & \\
\hline$(2,0)$ & $(2,0)$ & 384 & $2^{1+4} \times 2: S_{3}$ & regular \\
$(2,0)$ & $(3,0)$ & 1296 & $S_{3} \times S_{3} \times S_{3}: S_{3}$ & regular \\
$(2,0)$ & $(4,0)$ & 3072 & $2^{1+6}: 2^{2}: S_{3}$ & regular \\
$(2,0)$ & $(5,0)$ & 6000 & $5^{3}: 2: 2: 2: S_{3}$ & regular \\
$(2,0)$ & $(6,0)$ & 10368 & $2^{3}: S_{3}: S_{3}: S_{3}: S_{3}$ & regular \\
$(3,0)$ & $(1,1)$ & 144 & $S_{3} \times 2^{2}: S_{3}$ & regular \\
$(6,0)$ & $(1,1)$ & 576 & $2^{4}: S_{3}: S_{3}$ & regular \\
\hline$(3,0)$ & $(1,3)$ & 58968 & $L_{2}(27): 3: 2$ & chiral \\
$(1,2)$ & $(1,2)$ & 2688 & $2^{6}: 7: 3: 2$ & chiral \\
$(1,2)$ & $(2,1)$ & 1008 & $L_{2}(7) \times 3: 2$ & chiral \\
$(1,2)$ & $(3,1)$ & 58968 & $L_{2}(27): 3 \times 2$ & chiral \\
\hline
\end{tabular}

TABLE 6. Known finite universal hypertopes of type (2) in Figure 1 , with $\mathbf{s}=(a, b)$ and $\mathbf{t}=(c, d)$

by the correspondence $\alpha_{0} \mapsto \rho_{0}, \alpha_{1} \mapsto \rho_{1}, \alpha_{2} \mapsto \rho_{2}$ and $\alpha_{3} \mapsto \rho_{1} \rho_{3}$. We obtain a C-group with the following diagram and the extra relations $\left(\alpha_{0} \alpha_{1} \alpha_{2} \alpha_{1} \alpha_{3} \alpha_{2}\right)^{2 s}$ and $\left(\alpha_{1} \alpha_{2} \alpha_{3}\right)^{4}=1$.

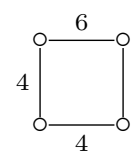

Now if we take the index 2 subgroup of $G,\left\langle\alpha_{1}^{\alpha_{0}}, \alpha_{1}, \alpha_{2}, \alpha_{3}\right\rangle$, we obtain a nonlinear hexagonal extention of the tetrahedron, with residues $\{3,6\}_{(2,0)}$ and $\{3,6\}_{(s, 0)}$ and order $48 s^{3}$. In summary, these hypertopes are constructed from $\{4,3,4\}_{(s, s, 0)}$ using a Petrie operation and then doubling the fundamental region of the Petrial.

It is interesting to see how we can obtain a permutation representation of the group of these locally toroidal hypertopes combining the permutation representation graphs of $\{3,6\}_{(2,0)}$ and $\{3,6\}_{(s, 0)}$. For a better understanding about permutation representation graphs of polytopes, called CPR graphs see [35]. Let us first consider the case $s$ even. We claim that the permutation representation graph of $\{3,6\}_{(s, 0)}$ is

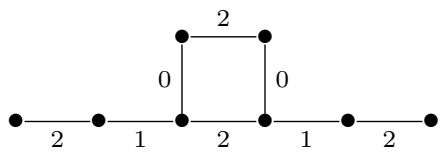

when $s=2$,

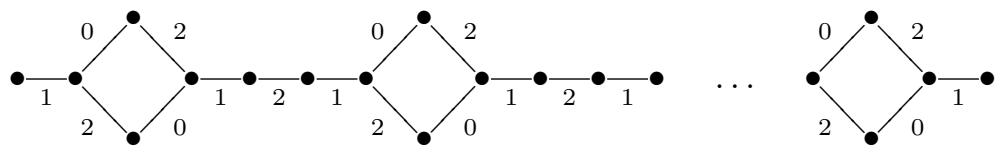

when $s$ is even and $s \geq 4$, where the number of alternating squares of the permutation representation graph is $s / 2$. Let $\alpha=\rho_{0} \rho_{1} \rho_{2} \rho_{1} \rho_{2} \rho_{1}$. To show that $\alpha^{s}=1$ observe that $\alpha$ acts as a translation on the vertices of the permutation representation graph such that $\alpha^{s}$ fixes all vertices of the permutation representation graph 
(see the following figure where $s=6$ ).

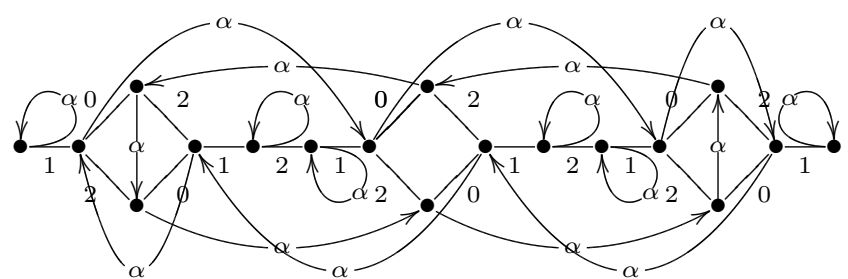

The size of the automorphism group of $\{3,6\}_{(s, 0)}$ is $12 s^{2}$. Let us prove that $12 s^{2}$ is also the size of $\left\langle\rho_{0}, \rho_{1}, \rho_{2}\right\rangle$. Consider the first point $x$ on the left of the permutation representation graph above. The group generated by $\alpha, \rho_{0}$ and $\rho_{2}$ is in the stabiliser of $x$ and has order $4 s$ (as $\alpha^{\rho_{0}}=\alpha^{-1}, \alpha^{\rho_{2}}=\alpha$ and $\alpha_{0}$ commute with $\alpha_{2}$ ). The permutation representation graph has $3 s$ vertices and $\left\langle\rho_{0}, \rho_{1}, \rho_{2}\right\rangle$ acts transitively on it. Hence $\left|\left\langle\rho_{0}, \rho_{1}, \rho_{2}\right\rangle\right| \geq 4 s \cdot 3 s=12 s^{2}$. Hence the graph above is a permutation representation graph of the automorphism group of $\{3,6\}_{(s, 0)}$.

When $s$ is odd it can be shown that the permutation representation graph of $\{3,6\}_{(s, 0)}$ is as follows.

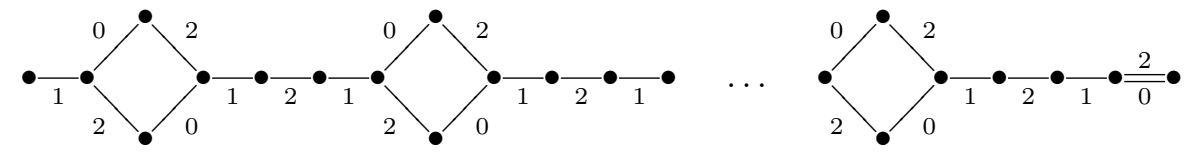

The number of alternating squares of the permutation representation graph is $\frac{s-1}{2}$. The proof that this graph is a permutation representation graph of the automorphism group of $\{3,6\}_{(s, 0)}$ when $s$ is odd is similar to the proof in the case $s$ even.

To obtain the permutation representation graph of the infinite family of locally toroidal hypertopes with residues $\{3,6\}_{(2,0)}$ and $\{3,6\}_{(s, 0)}$ we combine the respective permutation representation graphs and we obtain the following graphs when $s \geq 3$ accordantly as if $s$ is even or odd.
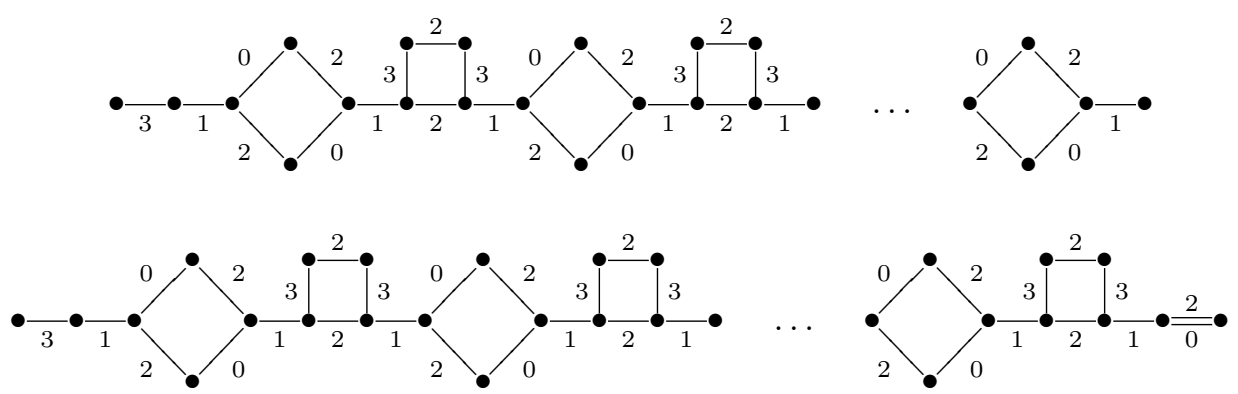

To prove that this is the group $G$ generated by the permutations $\rho_{0}, \rho_{1}, \rho_{2}$ and $\rho_{3}$ and that this group has order $48 s^{3}$, consider the vertex $x$ on the left in each of the permutation representation graphs. The stabilizer of $x$ contains the group generated by $\rho_{0}, \rho_{1}$ and $\rho_{2}$, of size $12 s^{2}$, and $G$ is transitive on the $4 s$ vertices of the graph. Thus $|G| \geq 48 s^{3}$.

Observe that lines 6 and 7 of Table 6 might suggest there could be a similar infinite family of regular locally toroidal hypertopes with diagram having toroidal rank 3 residues $\{3,6\}_{(1,1)}$ and $\{3,6\}_{(s, 0)}$ with $s \equiv 0$ mod 3 . A check with MaGmA 


\begin{tabular}{|c|c|c|c|c|c|}
\hline$p$ & $\mathbf{s}$ & $\mathbf{t}$ & $\# G$ & $G$ & \\
\hline 3 & $(3,0)$ & $(1,1)$ & 360 & $A_{5}: S_{3}$ & regular \\
& $(6,0)$ & $(1,1)$ & 23040 & $2^{4}: A_{5}: 2: 2: S_{3}$ & regular \\
& $(1,2)$ & $(1,1)$ & 1512 & $L_{2}(8): 3$ & chiral \\
& $(1,4)$ & $(1,1)$ & 90720 & $L_{2}(8) \times A_{5}: 3$ & chiral \\
& $(2,4)$ & $(1,1)$ & 774144 & $2^{9} \cdot L_{2}(8): 3$ & chiral \\
\hline 4 & $(2,0)$ & $(1,1)$ & 4320 & $A_{6}: 2 \times S_{3}$ & regular \\
& $(1,1)$ & $(1,1)$ & 3456 & $2^{1+4}: 3: S_{3}: S_{3}$ & regular \\
\hline
\end{tabular}

TABLE 7. Known finite universal hypertopes of type (3) in Figure 1 with $p=3,4, \mathbf{s}=(a, b), \mathbf{t}=(c, d)$

shows that the same groups appear in that case for $s \equiv 0 \bmod 6$ and for $s \equiv 3$ $\bmod 6$ with $s<100$.

\section{4-CIRCUITS WITH HEXAGONAL RESIDUES}

We now consider a hypertope $\mathcal{H}$ with the following B-diagram, where $x, y$ and $z$ are rotations generating the corresponding $\mathrm{C}^{+}$-group and $p=3,4,5,6$.

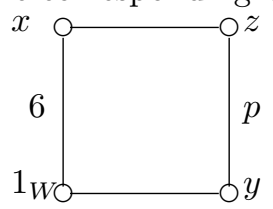

In $\mathcal{H}$ the two type $\{3,6\}$ residues may be nonisomorphic. Thus when $p=3,4$ or 5 , we need four parameters $a, b, c, d$ giving two additional relations in the following presentation for the the rotation subgroup of $G:=\operatorname{Aut}(\mathcal{H})$. The automorphism groups of the hypertopes of Table 7 are obtained using the following presentation where $\mathbf{s}=(a, b)$ and $\mathbf{t}=(c, d)$.

$$
\begin{gathered}
G^{+}(a, b, c, d):=\langle x, y, z| x^{6}, y^{3}, z^{2},(z x)^{3},(z y)^{p},\left(x^{-1} y\right)^{2}, \\
\left.\left(y^{-1} x^{-2}\right)^{a}\left(y x^{2}\right)^{b},\left(z x^{3}\right)^{c}\left(x^{-1} z x^{-2}\right)^{d}\right\rangle .
\end{gathered}
$$

Observe that no chiral universal hypertope was found for $p=4$ with $\mathbf{s}, \mathbf{t} \in$ $\{\{s, 0\},\{s, s\},\{s, t\} \mid s, t \in\{1, \ldots, 6\}\}$. Observe also that no universal hypertope (either regular or chiral) was found for $p=5$ with $\mathbf{s}, \mathbf{t} \in\{\{s, 0\},\{s, s\},\{s, t\} \mid s, t \in$ $\{1, \ldots, 6\}\}$.

When $p=6$, we need eight parameters $a, b, c, d, e, f, g, h$ giving four additional relations in the following presentation for the the rotation subgroup of $G:=\operatorname{Aut}(\mathcal{H})$. The automorphism groups of the hypertopes of Table 8 are obtained using the following presentation where $\mathbf{s}=(a, b), \mathbf{t}=(c, d), \mathbf{u}=(e, f)$ and $\mathbf{v}=(g, h)$.

$$
\begin{gathered}
G^{+}(a, b, c, d, e, f, g, h):=\langle x, y, z| x^{6}, y^{3}, z^{2},(z x)^{3},(z y)^{6},\left(x^{-1} y\right)^{2}, \\
\left.\left(y^{-1} x^{-2}\right)^{a}\left(y x^{2}\right)^{b},\left(z x^{3}\right)^{c}\left(x^{-1} z x^{-2}\right)^{d},\left(x^{-1} z\left(y^{-1} z\right)^{2}\right)^{e}\left(z x(z y)^{2}\right)^{f},\left(y(z y)^{2}\right)^{g}\left(y^{-1}(y z)^{2}\right)^{h}\right\rangle .
\end{gathered}
$$

We give several universal hypertopes with the help of Magma. These let us conjecture that two infinite families of finite locally toroidal hypertopes arise.

For each integer $s \geq 1$, the quotient $T_{s}$ of the Coxeter group $[3,3,4,3]$ with diagram 


\begin{tabular}{|c|c|c|c|c|c|c|}
\hline $\mathbf{s}$ & $\mathbf{t}$ & $\mathbf{u}$ & $\mathbf{v}$ & $\# G$ & $G$ & \\
\hline$(2,0)$ & $(1,1)$ & $(s, s)$ & $(2,0)$ & $1152 s^{4}$ & $s^{4}: 2^{1+4}: S_{3}: S_{3}$ & regular \\
$(2,0)$ & $(2,0)$ & $(1,1)$ & $(3,0)$ & 720 & $A_{5} \times 2: S_{3}$ & regular \\
$(2,0)$ & $(2,0)$ & $(1,1)$ & $(6,0)$ & 46080 & $2^{4}: A_{5}: 2 \times 2: 2: S_{3}$ & regular \\
$(2,0)$ & $(1,1)$ & $(4,0)$ & $(3,0)$ & 165888 & $2^{1+6}: S_{3}: S_{3}: S_{3}: S_{3}$ & regular \\
$(3,0)$ & $(2,0)$ & $(1,1)$ & $(2,0)$ & 1296 & $S_{3} \times S_{3} \times S_{3}: S_{3}$ & regular \\
$(3,0)$ & $(2,0)$ & $(1,1)$ & $(3,0)$ & 13824 & $2^{1+6}: 3: S_{3}: S_{3}$ & regular \\
$(3,0)$ & $(2,0)$ & $(1,1)$ & $(4,0)$ & 165888 & $2^{1+6}: S_{3}: S_{3}: S_{3}: S_{3}$ & regular \\
$(3,0)$ & $(2,0)$ & $(1,1)$ & $(5,0)$ & 5184000 & $2 \cdot\left(A_{5} \times\left(A_{5} \times A_{5}\right)\right): 2: S_{3}$ & regular \\
$(2,0)$ & $(1,2)$ & $(1,1)$ & $(6,0)$ & 15120 & $3 \times S_{7}$ & chiral \\
$(2,0)$ & $(1,2)$ & $(1,2)$ & $(2,0)$ & 352800 & $L_{2}(49) \times 3: 2$ & chiral \\
$(2,0)$ & $(1,2)$ & $(1,1)$ & $(0,3)$ & 7620480 & $S_{7} \times\left(L_{2}(8): 3\right)$ & chiral \\
\hline
\end{tabular}

TABLE 8. Known finite universal hypertopes of type (3) in Figure 1 with $p=6, \mathbf{s}=(a, b), \mathbf{t}=(c, d), \mathbf{u}=(e, f), \mathbf{v}=(g, h)$

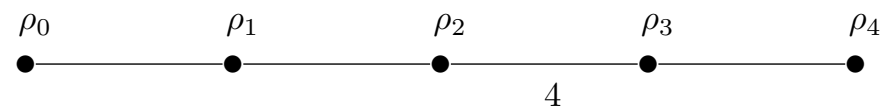

and additional relations

$$
\left(\rho_{0} \rho_{1}\left(\rho_{2} \rho_{3} \rho_{4}\right)^{3}\right)^{2 s}=1_{W}
$$

is the automorphism group of the rank 5 toroid $\{3,3,4,3\}_{(s, 0,0,0)}$ (see [30, Section $6 \mathrm{E}])$. The subgroup of $T_{s}$ generated by

$$
\tau_{0}:=\rho_{1}, \tau_{1}:=\rho_{2}, \tau_{2}:=\rho_{1} \rho_{3}, \tau_{3}:=\rho_{0} \rho_{4}
$$

is the automorphism group of the regular hypertope with diagram

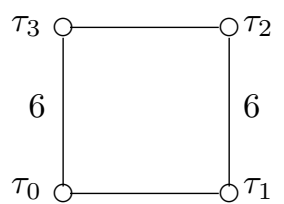

In fact, $\rho_{4}=\left(\tau_{0} \tau_{3}\right)^{3}$ and therefore $\rho_{0}=\left(\tau_{0} \tau_{3}\right)^{2} \tau_{0}$ yielding that $T_{s}=\left\langle\tau_{0}, \tau_{1}, \tau_{2}, \tau_{3}\right\rangle$. This suggests a correspondence between $\{3,3,4,3\}_{(s, 0,0,0)}$ and the infinite family of finite hypertopes mentioned in the first line of Table 8

Lines 5 to 8 of Table 8 also suggest the existence of an infinite family of finite hypertopes but we are unable to conjecture what will be the size of the automorphism group and what will be its structure. This is Problem 9.2 included in the next section.

\section{Future WORK AND OPEN PROBLEMS}

The basic theory of highly symmetric hypertopes was recently established in [21] but very few universal hypertopes were given. This paper, in a way, supplements it with numerous particularly interesting universal hypertopes. In each case, given a B-diagram and preassigned residues we establish the existence of the corresponding universal hypertope by checking the conditions established in [21]. Extensions of regular or chiral maps $\{4,4\}_{(b, c)}$ will give a hypertope whose residues are not all either spherical or toroidal. For instance, $\left\{\{4,4\}_{(4,0)},\{4,6\}_{3}\right\}$ gives a finite group 
of order 768 . The $\{4,6\}_{3}$ is non-orientable of genus 4 . We decide not to study this case here but this case is definitely interesting for future research. Other similar hexagonal extensions include $[3,6,6]$ and a star diagram with labels $(4,4,6)$.

We conclude this paper with some open problems and conjectures.

Problem 9.1. Can Theorem 2E17 of [30] be generalised to regular and chiral hypertopes?

Problem 9.2. Determine whether or not lines 5 to 8 of Table 8 are part of an infinite family of hypertopes with $(\mathbf{s}, \mathbf{t}, \mathbf{u}, \mathbf{v})=((3,0),(2,0),(1,1),(s, 0))$ with $s \geq 2$ an integer.

Conjecture 9.1. Table 3 gives a complete list of finite universal chiral polytopes of type $\{6,3,6\}$.

Conjecture 9.2. There are no finite universal chiral hypertopes with the following diagram.

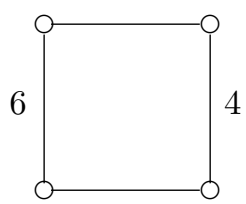

Conjecture 9.3. There is no finite universal regular or chiral hypertope with the following diagram.

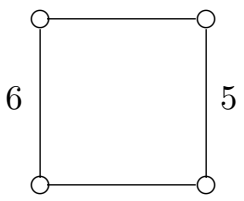

\section{ACKNOWLEDGEMENTS}

The authors would like to thank two anonymous referees for their numerous helpful and insightful comments. This research was supported by a Marsden grant (UOA1218) of the Royal Society of New Zealand, by NSERC and by the Portuguese Foundation for Science and Technology (FCT-Fundação para a Ciência e a Tecnologia), through CIDMA - Center for Research and Development in Mathematics and Applications, within project UID/MAT/04106/2013.

\section{REFERENCES}

[1] Michael Aschbacher. Flag structures on Tits geometries. Geom. Dedicata 14(1):21-32, 1983.

[2] Francis Buekenhout. Diagrams for geometries and groups. J. Combin. Theory Ser. A 27(2):121-151, 1979.

[3] Francis Buekenhout. $\left(g, d^{*}, d\right)$-gons. In Finite geometries (Pullman, Wash., 1981), volume 82 of Lecture Notes in Pure and Appl. Math., pages 93-111. Dekker, New York, 1983.

[4] Francis Buekenhout, editor. Handbook of Incidence Geometry: Buildings and Foundations. Elsevier Science, North-Holland, 1995.

[5] Francis Buekenhout and Arjeh M. Cohen. Diagram Geometry. Related to classical groups and buildings. Ergebnisse der Mathematik und ihrer Grenzgebiete. 3. Folge. A Series of Modern Surveys in Mathematics [Results in Mathematics and Related Areas. 3rd Series. A Series of Modern Surveys in Mathematics], 57. Springer, Heidelberg, 2013. xiv+592 pp.

[6] Francis Buekenhout, Michel Dehon, and Dimitri Leemans. An Atlas of residually weakly primitive geometries for small groups. Mém. Cl. Sci., Coll. 8, Ser. 3, Tome XIV. Acad. Roy. Belgique, 1999. 
[7] Francis Buekenhout and Michel Hermand. On flag-transitive geometries and groups. Travaux de Mathématiques de l'Université Libre de Bruxelles, 1:45-78, 1991.

[8] Charles J. Colbourn and Asia Ivić Weiss. A census of regular 3-polystroma arising from honeycombs. Discrete Math. 50:29-36, 1984.

[9] Marston Conder and Peter Dobcsányi. Determination of all regular maps of small genus. J. Combin. Theory Ser. B 81:224-242, 2001.

[10] Marston Conder, Isabel Hubard and Tomaž Pisanski. Constructions for chiral polytopes. J. Lond. Math. Soc. (2), 77:115-129, 2008.

[11] Marston Conder's website. http://www.math.auckland.ac.nz/ conder/. Last accessed September 21, 2014.

[12] Thomas Connor, Sebastian Jambor, and Dimitri Leemans. C-groups of PSL $(2, q)$ and PGL $(2, q)$. J. Algebra, 427:455-466, 2015.

[13] J.H. Conway, R.T. Curtis, S.P. Norton, R.A. Parker, and R.A. Wilson, Atlas of Finite Groups. Oxford U.P., 1985.

[14] Harold S. M. Coxeter. Configurations and maps. Rep. Math. Colloq. 8:18-38, 1948.

[15] Harold S. M. Coxeter. Twisted honeycombs. Regional Conf. Ser. In Math., Amer. Math. Soc., Providence, RI, 1970.

[16] Harold S. M. Coxeter and William O. J. Moser. Generators and relations for discrete groups, volume 14 of Ergebnisse der Mathematik und ihrer Grenzgebiete [Results in Mathematics and Related Areas]. Springer-Verlag, Berlin, fourth edition, 1980.

[17] Harold S. M. Coxeter and G. C. Shephard. Regular 3-complexes with toroidal cells. J. Combinatorial Theory Ser. B 22(2):131-138, 1977.

[18] Ludwig Danzer and Egon Schulte. Regulare Inzidenzkomplexe I. Geom. Dedicata 13:295-308, 1982.

[19] Michel Dehon. Classifying geometries with CAYLEY. J. Symbolic Comput. 17(3):259-276, 1994.

[20] David Elliott, Dimitri Leemans and Eugenia O'Reilly-Regueiro. Chiral polyhedra and projective lines. Int. J. Math. Stat. 16(1):45-52, 2015.

[21] Maria Elisa Fernandes, Dimitri Leemans and Asia Ivić Weiss. Highly symmetric hypertopes. Aequationes Math. 90:1045-1067, 2016.

[22] Dietmar Garbe. Über die Regularen Zerlegungen geschlossener orientierbarer Flächen. J. Reine. Angew. Math. 237:39-55, 1969.

[23] Branko Grunbaum. Regularity of graphs, complexes and designs, in Problèmes Combinatoires et Théorie des Graphes, Coll. Internat. CNRS, No. 260, Orsay, 1977, pp. 191-197.

[24] Michael I. Hartley, Isabel Hubard and Dimitri Leemans. Two atlases of abstract chiral polytopes for small groups. Ars Math. Contemp. 5:371-382, 2012.

[25] Lothar Heffter. Ueber Metacyklische Gruppen und Nachbarcnfigurationem. Math. Ann. 50:261-268, 1898.

[26] Isabel Hubard and Dimitri Leemans. Chiral polytopes and Suzuki simple groups. In $R$. Connelly et al. (eds.), Rigidity and Symmetry, Fields Institute Communications 70:155-175, 2014.

[27] Dimitri Leemans and Jérémie Moerenhout. Chiral polyhedra arising from almost simple groups with socle PSL $(2, q)$. J. Algebraic Combin. 44:293-323, 2016.

[28] Dimitri Leemans and Laurence Vauthier. An atlas of abstract regular polytopes for small groups. Aequationes Math. 72:313-320, 2006.

[29] Peter McMullen and Egon Schulte. Locally toroidal regular polytopes of rank 4. Comment. Math. Helv. 67(1):77-118, 1992.

[30] Peter McMullen and Egon Schulte. Abstract regular polytopes, volume 92 of Encyclopedia of Mathematics and its Applications. Cambridge University Press, Cambridge, 2002.

[31] Peter McMullen and Egon Schulte. Locally Unitary groups and regular polytopes. Adv. in Appl. Math. 29(1):1-45, 2002.

[32] Barry Monson and Asia Ivić Weiss. Eisenstein integers and related C-groups. Geom. Dedicata 66, 99-117, 1997.

[33] Barbara Nostrand and Egon Schulte. Chiral polytopes from hyperbolic honeycombs. Discrete Comput. Geom. 13:17-39, 1995.

[34] Antonio Pasini. Diagram Geometries. Oxford Science Publications, Oxford, 1994.

[35] Daniel Pellicer. CPR graphs and regular polytopes. European J. Combin. 29(1): 59-71, 2008. 
[36] Daniel Pellicer. A construction of higher rank chiral polytopes. Discrete Math. 310:1222-1237, 2010.

[37] Egon Schulte and Asia Ivić Weiss. Chiral polytopes. In Applied geometry and discrete mathematics, volume 4 of DIMACS Ser. Discrete Math. Theoret. Comput. Sci., pages 493-516. Amer. Math. Soc., Providence, RI, 1991.

[38] Egon Schulte and Asia Ivić Weiss. Chirality and projective linear groups. Discrete Math. 131, 221-261, 1994.

[39] Egon Schulte and Asia Ivić Weiss. Free extensions of chiral polytopes. Canad. J. Math. 47:641-654, 1995.

[40] Frank A. Sherk. A family of regular maps of type \{6,6\}. Canad. Math. Bull. 5:13-20, 1962.

[41] Duncan M. Y Sommerville. An Introduction to the Geometry of $n$ Dimensions. Methuen, London, 1929.

[42] Jacques Tits. Sur les analogues algébriques des groupes semi-simples complexes. Colloque d'algèbre supérieure, tenu à Bruxelles du 19 au 22 décembre 1956, Centre Belge de Recherches Mathématiques, 261-289, Établissements Ceuterick, Louvain; Librairie Gauthier-Villars, Paris, 1957.

[43] Jacques Tits. Buildings of Spherical Type and Finite BN-Pairs. Springer Verlag Berlin Heidelberg New-York, 1974.

[44] T. R. S. Walsh. Hypermaps versus bipartite maps. J. Combinatorial Theory Ser. B, 18(2):155-163, 1975.

[45] Constantin Weber and Herbert Seifert. Die beiden Dodekaederräume. Mat. Z. 37:237-253, 1933.

Maria Elisa Fernandes, Center for Research and Development in Mathematics and Applications, Department of Mathematics, University of Aveiro, Portugal

E-mail address: maria.elisa@ua.pt

Dimitri Leemans, Department of Mathematics, University of Auckland, Private Bag 92019, Auckland 1142, New Zealand and Département de Mathématique, C.P. 216 Algèbre et Combinatoire, Université Libre de Bruxelles, Boulevard du Triomphe, 1050 Brussels, Belgium

E-mail address: dleemans@ulb.ac.be

Asia Ivić Weiss, Department of Mathematics and Statistics, York University, Toronto, Ontario M3J 1P3, Canada

E-mail address: weiss@mathstat.yorku.ca 